\title{
Primary Malignant Melanoma of Jejunum with Perforation Peritonitis: A Case Report
}

\author{
Authors \\ Vivek Vasudeo $^{1}$, Madhu Smita ${ }^{2}$, Nishant ${ }^{1}$, Shital Malua ${ }^{1}$ \\ ${ }^{1}$ Deparment of Surgery, Rajendra Institute of Medical Sciences, Ranchi, Jharkhand, India \\ ${ }^{2}$ Department of Radiodiagnosis, All India Institute of Medical Sciences, New Delhi, India \\ Corresponding Author \\ Vivek Vasudeo \\ Email: vasudeo.vivek@gmail.com, Mobile No.+919931704627
}

\begin{abstract}
Neuroendocrine tumours (NETs also known as carcinoid tumours), adenocarcinoma, GIST, lymphoma are the common malignant neoplasms of small bowel in that order. Malignant melanomas are the rare entities. They are found mostly as metastasis to small bowel. Primary malignant melanoma of small bowel is very rare with paucity of published reports. It can remain asymptomatic for long duration and is often diagnosed at an advanced stage or as in this case with perforation where no standardised management protocol is developed. The pathophysiology is debatable. It is currently challenging to differentiate primary and secondary gastrointestinal melanomas. In this case report, we have reported a case of primary malignant melanoma of jejunum presenting with perforation peritonitis. Laparotomyrevealed ablack, pigmented, ulceroproliferative jejunal massperoperatively. It was further confirmed on histopathology and immunohistochemistry as Malignant Melanoma. After ruling out the differentials and on retrograde specific clinical history and examination, the diagnosis of primary malignant melanoma of jejunum was made.

Keywords- primary malignant melanoma, jejunum, immunohistochemical, Gastrointestinal stromal tumour (GIST), metastasis, leiomyosarcomaand criteria.
\end{abstract}

\section{Introduction}

Primary malignant melanoma in gastrointestinal tract has been controversial to differentiate from metastatic melanoma especially because primary cutaneous lesions have the potential to regress and disappear. ${ }^{[1]}$ Moreover, it greatly resembles other neoplastic conditions that may raise a major diagnostic challenge of its existence. Malignant melanoma is a rare disease and account $1-3 \%$ of all malignant tumors of gastrointestinal tract. ${ }^{[2]}$ Most of these lesions have a primarymostly arising from skin, anorectum, retina and under the nails.
However, exact incidence of primary malignant melanoma arising de novo in the gastrointestinal tract is still unknown. We report a primary malignant melanoma in the jejunum managed in a tertiary care centre.

\section{Case Report}

A 48 years non diabetic, non-hypertensive male presented in the emergency department with complaints of acute onset pain and distension of abdomen of five days duration. He also did not pass stool or flatus since two days. He had no history of 
fever or trauma. He had no history of any chronic medical illness. Patient was married, non-alcoholic, mixed vegetarian and not allergic to known drug or food substance. On physical examination, patient was conscious, oriented but ill looking and dehydrated. Pulse rate was 116 per minute, blood pressure $116 / 82$ and respiratory rate $24 / \mathrm{min}$. The basic haematological and biochemical profile were within normal limits. X-Ray chest with abdomen was done. Gas under the diaphragm was seen. On local examination, abdomen was distended, diffusely tender with board like rigidity. Perforation peritonitis was the provisional diagnosis on basis of which the patient was taken for emergency exploratory laparotomy through midline incision. 4 litres of bilious fluid was aspirated and gut was found grossly contaminated and plastered. The intraoperative examination of the abdomen detailed a single, hard, pigmented, perforated mass in the proximal jejunum $5 \times 6 \mathrm{~cm}$ in dimensions projecting outside the lumen. No liver metastasis or mesenteric lymph node involvement was seen. Resection and anastomosis of the involved segment of jejunum was undertaken which included healthy bowel margins of $5 \mathrm{~cm}$ and wide excision of the mesentery. We made gastrointestinal stromal tumour and metastasis from any primary as the first two possible diagnosis respectively. The post-operative course was uneventful and the patient was discharged on sixth postoperative day. The surgical specimen was an ulcero-proliferative, deeply pigmented, exophytic growth with central necrosis and haemorrhage, non-circumferentially involving the layers of gut (Figure-1).The histological report highlighted malignant melanoma of the jejunum (Figure-4). Of all the subcentimetric lymph nodes resected, none were positive for malignancy. It was sent for immunohistochemical markers and was found immunoreactive, score 4+ in spindled and pleomorphic neoplastic cells for MELAN-A (A differentiation antigen expressed in $100 \%$ of melanocytes), immunoreactive for HMB-45 (A monoclonal antibody for malignant melanoma) and non immunoreactive for DOG-1 (A cell surface protein selectively expressed in gastrointestinal stromal tumours ) [Figure-2, 3].

On retrograde examination, scalp, skin, eyes, mucus membranes and anus were negative for primary melanoma. The patient did not give any past medical history of excision of pigmented skin lesions. Further, repeat ultrasound abdomen for liver, contrast enhanced CT head, chest and abdomen were done and they did not report any metastasis or primary to this lesion. The patient has completely recovered and there is no evidence of metastasis to any other site since the past twelve months. We made the final diagnosis as primary melanoma of small intestine.

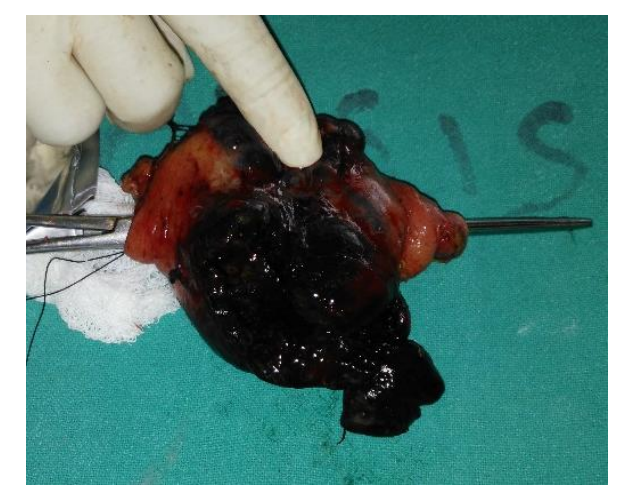

Fig 1 Resected specimen of jejunum showing pigmented, perforated jejunal mass.

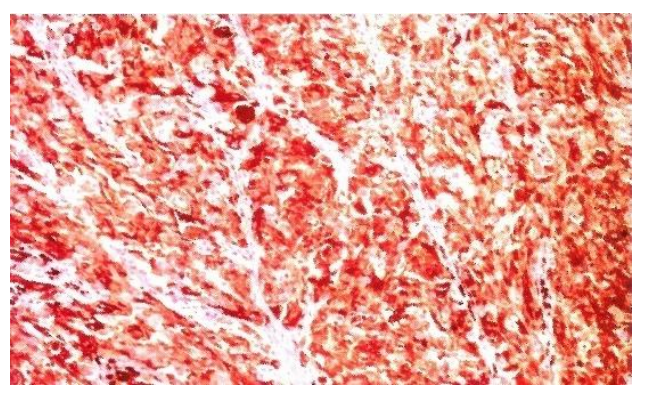

Fig 2 Immunostaining for Melan A : Positive

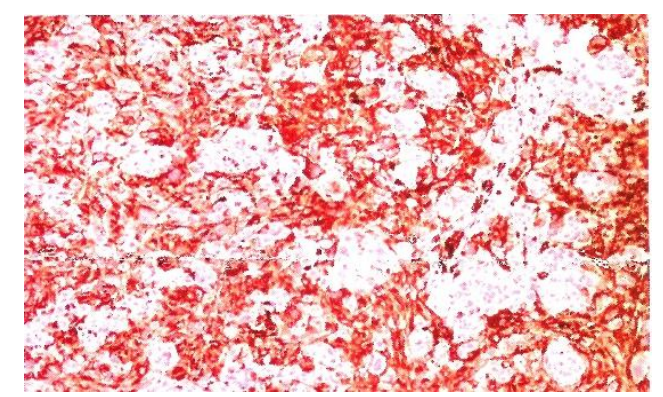

Fig 3 Immunostaining for HMB 45 : Positive 


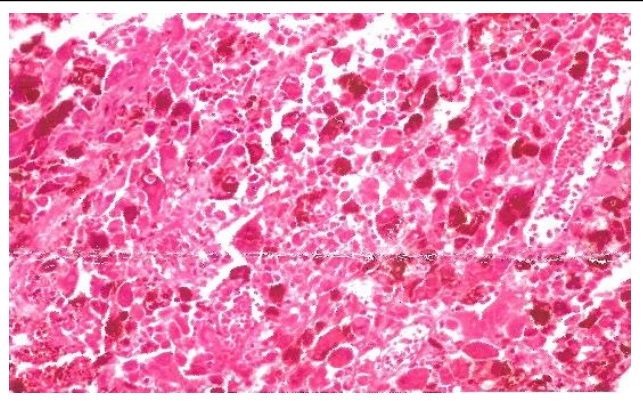

Fig 4: Hematoxylin and Eosin stain 400x: Loose arrangement of melanoma cells with melanin deposits

\section{Discussion}

Malignant melanoma of small bowel are generally asymptomatic. When symptomatic, they can present as chronic abdominal pain, occult or gross bleeding and weight loss. Sometimes it may present in the emergency with intestinal perforation which is very rare. Since, the presentations are varied, any exophytic mass lesion of jejunum with no history of obstruction, first differential is gastrointestinal stromal tumour (GIST) followed by metastasis from any primary, adenocarcinoma, neuroendocrine tumours and lymphoma.

GIST are mesenchymal tumours and represent $9 \%$ of all small bowel tumours. Jejunum forms the second most common site after stomach. In small bowel they are more often malignant than in stomach with $50 \%$ having metastasis at time of diagnosis to liver or peritoneum. Malignant GIST predominantly grow extraluminally and can show necrosis, haemorrhage and fistula formation. Obstruction is rare because GIST do not involve a circumferential bowel wall. Lymph node metastasis is also not commonly seen. Metastasis is more common malignancy in small intestine than primary tumour with nonspecific clinical manifestations of nausea, vomiting, abdominal pain and melena. It can involve duodenum, jejunum and distal ileum. ${ }^{[3]}$ Typical presentation of adenocarcinoma is focal unilocular circumscribed mass with shouldering of the margins and obstruction. Ulceration is quite common. Metastasis to liver and peritoneum occurs frequently. Primary small bowel lymphoma rarely cause obstruction, however, it is a less common cause of perforation. It typically shows asymme- trical but circumferential wall thickening with associated luminal dilatation which in contrary to our case show deeply pigmented focal ulceroproliferative growth. Benign lesions like leiomyoma may mimic this condition however large ulceroproliferative mass more than $6 \mathrm{~cm}$ have extraluminal should raise suspicion of leiomyosarcoma. ${ }^{[3,4]}$ Small bowel leiomyosarcoma may occur anywhere in the small bowel, mainly ileum and jejunum. Absence of irregular lesion margins and enlarged lymph nodes downgrade its possibility as a differential diagnosis.

Primary malignant melanoma of bowel being a rare entity is the diagnosis of exclusion after ruling out primary at any other site. Metastatic melanoma from a known primary has been well documented. Mostly they arise from skin. Primary malignant melanoma of bowel might arise from schwannian neuroblast cells associated with autonomic innervations of gut. ${ }^{[5]}$ Some postulate that neural crest cells migrate into the gut. Mature melanocytes in gut is rare, therefore they have an idea that occult or regressed primary is always the original source, spontaneous regression of cutaneous melanoma can occur. ${ }^{[6]}$ Since several cases of the gastrointestinal tract tumour have been reported as primary malignant melanoma and followed up, different diagnostic criteria's have been put forward by different people of thought. To summarise them, first criteria for primary malignant melanoma is absence of other primary site melanoma and no history of removal of melanoma or atypical melanocytic lesions from skin, retina anal canal and occasionally at other locations like oesophagus, penis or vagina. ${ }^{[2,7]}$

Second criteria established by Sacks et al. [8] included three parameters which are 1) Presence of single lesion 2) Other organs free of primary lesions and absence of enlargement of draining lymph nodes and 3) Survival time more than one year after the diagnosis.

Blecker et al. ${ }^{[9]}$ proposed a new criteria for the diagnosis of primary melanoma of the small bowel which also included three parameters 1) Presence of solitary mucosal lesion in the intestinal epithelium 2) 
Absence of melanoma or atypical melanocytic lesions of the skin. 3) Presence of intramucosal melanocytic lesions in the overlying or adjacent intestinal epithelium.

Histologic criteria is based on the proliferation of atypical junctional melanocytes and atypical melanocytic cells in the basal layer of the superficial epithelium. Other indications of primary lesions are the presence of lymphocytic infiltration surrounding the tumour mass. ${ }^{[9,10]}$

Our case satisfies all the above diagnostic criteria. Acute abdomen caused by small bowel perforation due to metastatic malignant melanoma is extremely rare condition. Less than 20 cases have been published till date. However reporting of primary malignant melanoma presenting with perforation is in counts of one or two. Reporting such a rare case may provide data for management of similar cases till date.

\section{Conclusions}

Primary malignant melanoma, though is a rare possibility but we must be aware of its occurrence and can be kept as one of the differential of a patient presenting with acute abdomen with perforation in emergency and having pigmented ulcero-proliferative mass in small bowel on peroperative examination. Since the prognosis of the individual is good with a long life expectancy, reporting of these cases must be done to gain a standardisation of treatment.

\section{References}

1. R. J. Bramhall, K. Mahady, A. H. Peach, "Spontaneous regression of metastatic melanoma - clinical evidence of the abscopal effect," Eur J SurgOncol.,vol 40, pp. 34-41, 2014.

2. K. S.Atmatzidis, T. E. Pavlidis, B. T. Papaziogas, "Primary malignant melanoma of the small intestine: report of a case," Surg Today,vol 32, no. 9, pp. 831-833, 2002.
3. E. J. Rummeny, P. Reimer, W. Heindel,"MR Imaging of the Body,"Thieme Medical Pub.(2009) ISBN:3131358416

4. L. Baert, "Encyclopedia of Diagnostic imaging, "Springer Verlag(2007) ISBN: 3540352783

5. Y. Mishima, "Melanocytic and nevocytic malignant melanomas. Cellular and subcellular differentiation,"Cancer, vol. 20, pp. 632-649, 1967.

6. M. Elsayed, M. Albahra,U. C. Nzeako, L. H.Sobin, "Malignant melanomas in the small intestine: a study of 103 patients," Am J Gastroenterol., vol. 91, no. 5, pp. 1001-1006, May 1996.

7. S. Kruger, F. Noack, C. Blochle, A. C. Feller, "Primary malignant melanoma of the small bowel: a case report and review of the literature,'Tumori, vol. 91, no. 1, pp.73-76, 2005.

8. D. L.Sachs, L. Lowe, A. E. Chang, E. Carson, T. M. Johnson, "Do primary small intestinal melanoma exist? Report of a case,'J Am Acad Dermatol, vol. 41, pp. 1042-1044,1999.

9. D. Blecker, S. Abraham, E. E. Furth, Kochman,"Melanoma in the gastrointestinal tract,"Am J Gastroenterol., vol. 94, no. 12, pp. 3427-3433, 1999.

10. T. K.Timmers, E. M. Schadd, J. F. Monkelbaan, et al, "Survival after resection of a primary malignant melanoma of the small intestine in a young patient: report of a case," Case Rep Gastroenterol., vol. 7 no. 2,pp.251-260, May-Aug. 2013. 